\title{
Expert Opinions on the Debate of Pericardial Reconstruction in Cardiovascular Surgery: To Close or Not to Close?
}

\author{
Alfredo Rego, MD, PhD, ${ }^{1}$ W. Douglas Boyd, $\mathrm{MD},{ }^{2}$ Enrique Gongora, $\mathrm{MD},{ }^{3}$ William E. Johnson 3rd, $\mathrm{MD},{ }^{4}$ \\ Nabil A. Munfakh, MD, John Pirris, MD, ${ }^{6}$ Randall K. Wolf, MD $^{7}$ \\ ${ }^{1}$ Jackson Heart Institute, Department of Cardiothoracic Surgery, Jackson Health System, Miami, Florida; \\ ${ }^{2}$ Division of Cardiothoracic Surgery, Department of Cardiovascular Sciences, Brody School of Medicine, East Carolina University, \\ Greenville, North Carolina; ${ }^{3}$ Division of Cardiothoracic Surgery, Department of Surgery, University of Alabama at Birmingham \\ School of Medicine, Birmingham, Alabama; ${ }^{4}$ Infirmary Health, Cardio-Thoracic and Vascular Surgical Associates, Mobile, Alabama; \\ ${ }^{5}$ Division of Cardiothoracic Surgery, Department of Surgery, Washington University School of Medicine in St. Louis, St. Louis, \\ Missouri; ${ }^{6}$ Division of Cardiothoracic Surgery, Department of Surgery, University of Florida College of Medicine, Jacksonville, \\ Florida; ${ }^{7}$ DeBakey Heart and Vascular Center, Houston Methodist Hospital, Texas Medical Center, Houston, Texas
}

\section{ABSTRACT}

Background: As of 2019, pericardial closure was performed in only a small portion of the over 320,000 cardiac surgeries performed annually. However, evidence regarding the benefits of pericardial closure or reconstruction has been accruing, particularly with the publication of the RECON study in 2019.

Methods: This group of authors convened to try to arrive at consensus expert opinion regarding pericardial reconstruction. Structured topic questions initially were used to stimulate discussion. Subsequently, a survey of proposed expert opinion statements was conducted among the authors. Based on that survey, consensus expert opinion statements and recommendations were compiled.

Results: The expert opinions encompass various topics relating to pericardial reconstruction, including definitions, benefits/risks, and technique. Observed benefits include reductions in: (1) adhesions; (2) postoperative pericardial effusion, atrial fibrillation, and bleeding; and (3) readmissions and length of hospital stay. Expert opinion recommendations regarding surgical technique are compiled into a single chart. Complete pericardial reconstruction should be performed, using native pericardial tissue if available and viable; if not feasible, a patch may be used. Patches that stimulate the formation of site-specific tissue in situ (such as natural extracellular matrix) may have additional benefits (including bioregenerative properties and lack of inflammatory response). Closure should be taut, but tension-free. Adequate drainage of the closed pericardium must be ensured.

Received May 5, 2021; received in revised form September 10, 2021; accepted September 13, 2021.

Correspondence: Alfredo Rego, MD, PhD, 3650 N.W. 82nd Avenue, Suite 208, Miami, FL 33166, Telephone 486-466-8484, Fax 305-355-1560 (e-mail: Alfredo.Rego@jbsmiami.org).
Conclusions: Based on available data and collective surgical experience, we endorse pericardial reconstruction as standard approach in appropriately selected patients. We also endorse adoption of standardized pericardial reconstruction techniques to optimize patient outcomes and improve evidence quality in future studies.

\section{INTRODUCTION}

Following open heart surgery, such as coronary artery bypass graft (CABG) or valve repair/replacement surgery, surgeons may choose to leave the pericardium open or may opt for pericardial closure, also known as pericardial reconstruction (PCR). Because of postoperative swelling of cardiac tissues, some physicians prefer not to close the pericardium based on the perception that closure may compromise cardiac hemodynamics or be associated with pericardial tamponade. Other physicians endorse PCR to preserve the natural anatomy and physiology of the heart, to minimize adhesions, or for a variety of other reasons, such as to maintain retrosternal space and prevent right ventricular (RV) contact with the posterior sternal table.

Primary closure of the pericardium with the patient's native pericardial tissue following open heart surgery does not provide margin for postoperative myocardial edema, inflammation, and accumulation of fluid. In fact, studies [Damen 1989; Daughters 1992; Hunter 1992; Izzat 1994; Jarvinen 1987] have shown alterations in several cardiac parameters-cardiac output, cardiac index, stroke volume, right atrial, pulmonary arterial, and pulmonary capillary wedge pressures-in the early postoperative period following pericardial closure after open heart surgery. Using a synthetic or biosynthetic pericardial substitute in addition to or in place of native pericardium in PCR has the benefit, among others, of not causing immediate cardiac constriction.

Several studies have evaluated outcomes with pericardial closure or reconstruction, and Table 1 provides a summary of these studies. Table 1A displays randomized studies. Table 1B displays 
observational studies of 70 patients or more, and Table 1C displays studies which provide limited evidence based on methodology or small sample size. (Table 1A) (Table 1B) (Table 1C)

Several earlier studies [Jarvinen 1987; Damen 1989; Hunter 1992; Daughters 1992; Izzat 1994] focused almost exclusively on hemodynamic assessment of primary pericardial closure with invasive monitoring and echocardiography and found evidence of hemodynamic decline. Of historic interest, one of the earliest comparative observational studies [Nandi 1976] reported favorable clinical outcomes in 225 patients treated with pericardial closure compared with 596 patients in whom the pericardium was left open. The pericardial closure group required fewer reoperations and had a lower incidence of cardiac tamponade; among all patients requiring reoperation, there were lower rates of mortality, infection, and intubation observed among those who had been in the initial pericardial closure group. The majority of studies published since 1995 have utilized a synthetic or biosynthetic pericardial substitute to reconstruct the pericardium. Among studies published since 1995, most reported no increase in complications or a beneficial clinical outcome, such as fewer adhesions by CT scan [Duvernoy 1995], lower rates of postoperative atrial fibrillation (POAF) [Boyd 2010; Kaya 2015; Rego 2019] increased epicardial surface to posterior sternal table distance [Rao 1999], reduced infection [Kaya 2015], and fewer pericardial effusions [Kaya 2015; Rego 2019].

Table 1A. Randomized studies of pericardial reconstruction

\begin{tabular}{lll}
\hline $\begin{array}{l}\text { Author(s), date, journal, } \\
\text { country }\end{array}$ & $\begin{array}{l}\text { No. of } \\
\text { patients }\end{array}$ & Mean age, $y \quad$ Major findings
\end{tabular}

Asanza et al. [Asanza

200

1976], Ann Thorac Surg,

U.S.
[Duvernoy 1995], Thoracic Cardiovasc Surg, Sweden

Bhatnagar et al. [Bhatnagar 1998], J Card

Surg, Canada

Rao et al. [Rao 1999], Ann Thorac Surg,

Canada

Kaya et al. [Kaya 2015], Interact Cardiovasc

Thorac Surg, Turkey

142

Lower half of pericardium was approximated in midline with interrupted 3-0 silk sutures; if vein grafts were placed into the aorta, the upper portion of the pericardium was closed loosely using thymus on one side or approximating only thymic tissue over the vein grafts

$\mathrm{CABG} \pm$ valve; patients randomized to pericardial closure with biodegradable polyhydroxybutyrate patch vs. no pericardial closure

302

42

62

Isolated CABG membrane
Isolated CABG using Gore-Tex@ surgical

57.9 closed
vs. 55.9
open

First CABG with beta-blocker; used bilateral trap door incision, with insertion of intrapericardial drain, pericardio-pleural window, and total closure of pericardium
100 patients had closure and 100 were left open; alternate patients had the pericardium left open or closed (pseudo randomization). There was no significant difference between the two groups in postoperative stay, blood loss, or complications except that the closure group experienced more frequent pericardial rub ( $14 \%$ vs. $3 \%$ ). Incidence of post-pericardiotomy syndrome was equal. Two patients in the open group required re-exploration for bleeding. In patients who consented to postoperative angiography following revascularization procedures, there was no significant difference in the incidence of graft failure, which occurred in $9 \%$ of patients in the closure group vs. $13 \%$ of patients in the open group.

CT scan at 6 and 24 months postop showed a significantly lower incidence of pericardial adhesions between the patch and cardiac surface compared with the corresponding area in the non-patch group. A $27 \%$ reduction in patch size was also observed.

Multicenter study. 138 patients were closed with Gore-Tex membrane; pericardium was left open in 164. Fewer complications in Gore-Tex group, but not statistically significant. Two deaths occurred in each group.

20 patients had pericardial closure; 22 did not. Epicardial surface to posterior sternal table distance was greater postop in patients with closure. In the early postoperative period, the cardiac index and stroke work volume were lower in the closure group.

70 patients had pericardial closure; 72 "open" patients had partial pericardial closure. Atrial fibrillation occurred in $27.78 \%$ of patients with partial closure vs. $8.57 \%$ in patients with complete closure. On the 2 nd postoperative day, significantly fewer in closed group had (small) pericardial effusion. Lung infection developed in $11.1 \%$ of "open" group vs. $4.29 \%$ in closure group. Hospital and $\mathrm{CCU}$ length of stay was significantly shorter in patients in the closure group. 
Published in 2019, the prospective, observational RECON (A Post Market Observational Study to Obtain Additional Information on the Use of CorMatrix ECM for Pericardial Reconstruction) study evaluated PCR with extracellular matrix (ECM) in 1420 patients following open heart surgery [Rego 2019]. Most surgeries were CABG, valve replacement, or a combination of the two. Outcomes were compared with those from the National Readmissions Database (NRD) using inverse probability of treatment weighting to control for differences in demographics, comorbidities, and risk factors. In comparison to the NRD, the RECON outcomes favored PCR and showed statistically significant and clinically significant reductions in hospital readmissions, pleural effusion, pericardial effusion, and bleeding complications. Furthermore, POAF was far less common with PCR than in previously reported studies [Rego 2019].

As of 2019, pericardial closure or reconstruction was performed in only a small portion of the over 320,000 cardiac surgeries performed in the U.S. annually [Rego 2019]. Consensus was lacking among surgeons regarding whether or not

Table 1B. Observational studies of pericardial reconstruction (70 patients or more)

\begin{tabular}{|c|c|c|c|}
\hline $\begin{array}{l}\text { Author(s), date, journal, } \\
\text { country }\end{array}$ & $\begin{array}{l}\text { No. of } \\
\text { patients }\end{array}$ & Mean age, y & Methods \\
\hline $\begin{array}{l}\text { Cunningham et al. [Cun- } \\
\text { ningham 1975], J Thorac } \\
\text { Cardiovasc Surg, U.S. }\end{array}$ & 100 & - & $\begin{array}{l}\text { pericardium was kept under tension during } \\
\text { surgery to minimize shrinkage; intra- \& extra- } \\
\text { pericardial sumps were used }\end{array}$ \\
\hline
\end{tabular}

Nandi et al. [Nandi 1976], British Heart Journal, Hong Kong range 8.5 pericardium was closed in 225 and left open mos to 61 y in 596; primary pericardial closure performed with interrupted silk sutures, about $1.5 \mathrm{~cm}$ apart

range 1 mo PCR with Gore-Tex@ Surgical Membrane to $76 \mathrm{y}$ (SM)
Minale et al. [Minale Germany

Kargar and Aazami [Kargar 2007]; J Thorac Cardiovasc Surg, Iran
Boyd et al. [Boyd 2010], Heart Surg Forum, U.S. primary isolated CABG; PCR used porcinederived extracellular matrix patch (CorMatrix)

Rego et al. [Rego 2019], 1420 J Cardiothorac Surg, U.S.
100 patients had primary closure. Most postoperative bleeding originated from outside the pericardium. There were no instances of cardiac tamponade. However, 19 patients lost over $1 \mathrm{~L}$ of blood after surgery, and 5 required reoperation. Single-center, no control group. Unclear how patients were assigned to treatment of chest tubes.

Reoperation required in $6.87 \%$ open vs. $1.77 \%$ closed cases. Cardiac tamponade occurred in $3.85 \%$ open vs. $0 \%$ closed cases. Among reoperated patients, drainage amount was $36 \mathrm{ml}$ closed vs. $224 \mathrm{ml}$ open cases. Mortality for reoperations was $17.8 \%$ open vs. $0 \%$ closed cases. Tracheostomy was required in $22.22 \%$ of open reoperative cases vs. $0 \%$ in closed cases; wound infection occurred in $4(8.88 \%)$ of open reoperative cases and in only 1 closed reoperative case.

Mortality occurred in 3 patients. One episode of cardiac tamponade on 7th postoperative day. One patient developed mediastinal hematoma with fever and leukocytosis. Four patients required reoperation within 15 months, in these cases, there were no adhesions on the anterior wall with SM and the pericardium could be easily dissected.

One death due to stroke; no other major complications. No reoperations required and no post-pericardiotomy syndrome. At discharge, echocardiogram reveals no more than trivial pericardial effusion in any patient. At one month, one patient who had undergone mitral valve replacement required reoperation due to massive pericardial effusion associated with warfarin overdose.

Retrospective comparison with consecutive control group. 111 with PCR and 111 controls without closure. Postoperative atrial fibrillation occurred in $18 \%$ of PCR patients vs. $39 \%$ of control patients. Reduction in postoperative atrial fibrillation was $54 \%$.

RECON study. 923 CABG; 436 valve. In comparison with NRD cohort, 30-day readmission rate was significantly lower, and there were reduced rates of pleural effusion, pericardial effusion, and bleeding. Reduced rates of atrial fibrillation were observed in comparison with previous reports. 
Table 1C. Pericardial reconstruction studies providing limited evidence

\begin{tabular}{llll}
\hline $\begin{array}{l}\text { Author(s), date, } \\
\text { journal, country }\end{array}$ & $\begin{array}{c}\text { No. of } \\
\text { patients }\end{array}$ & Mean age, y & Methods \\
\hline
\end{tabular}

\begin{tabular}{|c|c|c|c|}
\hline $\begin{array}{l}\text { Jarvinen et al. [Jarvin- } \\
\text { en 1987], Scand J } \\
\text { Thorac Cardiovasc }\end{array}$ & 29 & - & $\begin{array}{l}\text { routine pericardial closure after cardiopulmo- } \\
\text { nary bypass in patients undergoing } C A B G \pm \\
\text { valve surgery }\end{array}$ \\
\hline urg, Finland & & & \\
\hline
\end{tabular}

Surg, Finland

\section{Damen [Da-}

men 1989]; Acta

30

Anaesthesiol Scand, Netherlands

Hunter et al. [Hunter 1992], Ann Thorac Surg, U.K.

patients undergoing CABG with normal LV function

59

Open heart valve surgery; observations made during surgery while pericardium open, and then closed. Repeat observations made
10

[Daughters 1992], J

Thorac Cardiovasc

Surg, U.S.
Izzat et al. [lzzat 1994], J Heart Valve Dis, U.K.
10

65 postop after pericardial suture had been removed 1.5-2 hours post-surgery

55.3 9 patients had CABG and 1 had ASD repair; pericardium was closed but then reopened 11-15 hours after surgery

tension free pericardial closure technique in patients undergoing open heart surgery

PCR was well tolerated clinically but resulted in $8 \%$ decrease in cardiac output. LV end diastolic cavity dimension decreased from 46 to $41 \mathrm{~mm}$. After pericardium was reopened, LV end diastolic cavity dimension increased to $45 \mathrm{~mm}$. Pericardial closure seemed to limit ventricular filling.

Closure of the pericardium resulted in statistically significant decreases in arterial blood pressure, cardiac index, mean RA, mean PA, and PCW pressures. The changes were likely caused by a change in the ventricular pressure-volume relationships.

Closing the pericardium caused an immediate and statistically significant drop in $\mathrm{CO}$ (thermodilution) of $1.39 \pm 0.24 \mathrm{~L} / \mathrm{min}$ from $5.09 \pm 0.40 \mathrm{~L} / \mathrm{min}$. Stroke volume decreased by $29 \%$ and SVR increased by $34 \%$. Opening the pericardium postop while the chest remained closed increased CO by $1.33 \pm 0.15 \mathrm{~L} / \mathrm{min}$ from $4.12 \pm 0.62 \mathrm{~L} / \mathrm{min}$. Stroke volume increased $15 \pm 3 \mathrm{~mL}$ from 53 $\pm 5 \mathrm{~mL}$ and SVR dropped by $473 \pm 83$ dyne.s.cm- 5 from $1721 \pm$ 181 dyne.s. $\mathrm{cm}-5$. The data suggest pericardial closure early after open heart surgery lowers stroke volume and cardiac output.

End-diastolic volume index, peak positive time derivative of pressure, stroke work index, and $\mathrm{Cl}$ all increased significantly when the pericardium was opened. Thus, the pericardium had a significant constraining effect on diastolic filling of the left ventricle. The improvement in LV systolic performance should be considered when contemplating pericardial closure in patients with preoperative LV dysfunction.

Observations were made during surgery, after closure with a tension free technique, and after chest closure. Observations at 1.5-2 hours postoperatively were made while the chest remained closed, both before and after the pericardium was reopened by removing the pericardial suture through the chest wall. PCR led to an $8 \%$ fall in cardiac output, a $15 \%$ fall in SVR, and a $13 \%$ drop in mean arterial pressure. Systolic and diastolic LV dimensions decreased by $6 \%$ and $4 \%$, respectively.

No complications related to the gelatin sheet were observed. At repeat surgery a mean of 1.4 years later, all surgeons rated the effectiveness of the gel as "good." The anti-adhesive prevented dense adhesions, suggesting the material may be useful as a pericardial substitute for multistage pediatric cardiac surgery.

30 patients had primary closure of pericardium vs 18 without; there were no complications or clinical implications; transient deterioration in hemodynamics was noted in those with primary closure.

ECM was used for pericardial closure in 4 patients and in 26 for cardiac tissue repair. No major complications over a follow-up period of 4-25 months.
ECM patch was used in surgical repair of congenital heart disease stage cardiac surgery; used novel biodegradable pericardial substitute developed from gelatin obtained from pathogen-free porcine skin and a bioabsorbable polyester mesh vs 59.6 continuous stitches

6.4 
Table 2. Functional goals of performing pericardial reconstruction ${ }^{a}$

\begin{tabular}{|c|c|}
\hline $\begin{array}{l}\text { Decrease adhesions/scarring for easier reentry [Duvernoy 1995; Matsumura } \\
\text { 2008; Minale 1988; Rego 2019] }\end{array}$ & Decrease tamponade [Nandi 1976] \\
\hline Avoid blood collection in pericardial well & Improve tube drainage after surgery [Nandi 1976] \\
\hline Infection protection [Kaya 2015; Nandi 1976] & More effective CPR when needed \\
\hline \multirow{3}{*}{ Reestablish normal physiology } & Restore asymmetrical RV constraint function \\
\hline & Prevent herniation of the heart into left chest \\
\hline & Compartmentalization to better identify sources of bleeding [Boyd 2012] \\
\hline
\end{tabular}

$\mathrm{CPR}$, cardiopulmonary resuscitation, $\mathrm{RV}$, right ventricle. ${ }^{\mathrm{a}} \mathrm{Goals}$ are not listed in any specific priority order.

Table 3. Data supporting PCR and reasons for positive RECON study results ${ }^{a}$

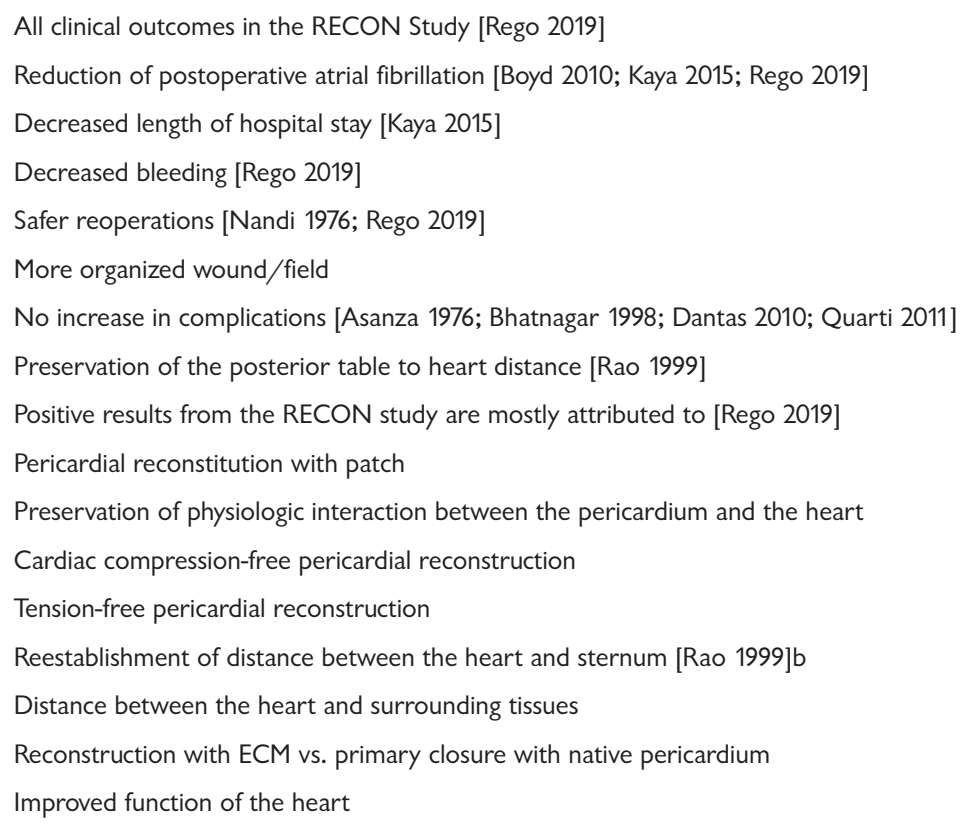

${ }^{a}$ not listed in any specific priority order; be.g., creating a barrier between the sternum and the heart ECM, extracellular matrix; PCR, pericardial reconstruction; RECON, A Post Market Observational Study to Obtain Additional Information on the Use of CorMatrix ECM for Pericardial Reconstruction

Table 4. Potential or actual risks of pericardial reconstruction ${ }^{\mathrm{a}}$

Kinking and/or compression of bypass conduits [Boyd 2012]

Cardiac tamponade secondary to active bleeding [Boyd 2012] and/or poor drainage

Myocardial compression leading to poor hemodynamics [Damen 1989; Dantas 2010; Hunter 1992; Izzat 1994; Jarvinen 1987; Rao 1999]

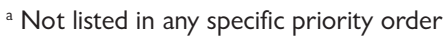


the pericardium should be closed or reconstructed; consensus also was lacking on optimal methods to repair the pericardium following cardiac surgery. Surgical approaches can differ, e.g., complete vs. partial reconstruction, whether to use a pericardial patch, which pericardial substitute to use, surgical and suturing techniques, and patient selection. Studies of PCR have incorporated a variety of surgical techniques, inclusion criteria, and evaluation endpoints, and many of the studies have significant limitations and/or risk of bias. As a result, high-quality evidence needed for the development of practice guidelines has been lacking.

This expert opinion report is a comprehensive analysis from the contributing authors, many of whom have years of experience and expertise in performing successful PCR. The intent of this communication is: 1) to provide recommendations for surgeons and trainees newly adopting PCR, and 2) to provide criteria for investigators assessing PCR efficacy and outcomes. Formal standardized guidelines regarding PCR could be developed once a sufficient body of high-quality evidence is available. Of course, the ultimate goal is to improve outcomes and quality of care in patients undergoing heart surgery.

\section{MATERIALS AND METHODS}

This group of authors consists of physicians, who regularly reconstruct the pericardium, and those who do not. Over the course of a few months, the group convened several times to discuss current techniques for PCR and to reach consensus regarding the techniques they would recommend to achieve optimal clinical outcomes. During a virtual forum held July 20, 2020 through August 11, 2020, structured topic questions were used to stimulate discussion on important issues related to PCR technique and patient outcomes. Techniques that had been unsuccessful, and lessons learned, also were discussed among the group. Following the virtual forum, a preliminary list of opinion-based statements and recommendations was compiled.

Subsequently, a written survey consisting of 30 survey items was conducted within the group between September 10, 2020 and September 21, 2020. The survey was divided into three sections: 1) the role, advantages, and risks of PCR, 2) patient selection for PCR, and 3) elements of surgical technique critical for clinical success of PCR.

For 28 of the survey questions, a proposed expert opinion statement was presented, and each workgroup member was asked to indicate if they were "aligned" or "not aligned" with the statement. Members could provide additional clarification or explanation along with their response(s). The workgroup leader would request further clarification or explanation from members of the group, if needed, to determine areas of agreement or disagreement with the proposed expert opinion statement(s), and members would respond with further explanation. One of the survey questions asked each workgroup member to rank the relative importance of items within the question. The final survey question was open-ended and solicited any additional or further comments or thoughts from each survey respondent.
Based on results of the survey, a list of expert opinion consensus statements and recommendations was compiled. All authors participated in generation of the expert opinion recommendations.

\section{RESULTS}

The expert opinion statements and recommendations developed by the group are described below.

\section{Section 1. The Role, Advantages, and Risks of Pericardial Reconstruction (PCR)}

The following are the proposed standardized definitions for PCR:

- Primary closure: Complete or partial closure using only native pericardial tissue.

- Complete reconstruction: The pericardium is fully closed with native pericardium and/or patch substitute to reestablish normal anatomy and function.

- Partial reconstruction: The pericardium is partially closed using native pericardium, thymic, and/or patch tissues in any location.

Partial reconstruction is not equivalent to complete reconstruction. However, partial reconstruction is not synonymous with "incomplete" reconstruction. Certain procedures, such as CABG with left internal thoracic artery (LITA) graft, may require partial reconstruction, yet the reconstruction is not "incomplete."

Goals of performing PCR: The functional goals of PCR are presented in Table 2. (Table 2) Evidence regarding protection against infection [Kaya 2015; Nandi 1976] may be weak at this time; however, PCR does act as a protective barrier [Rego 2019], in the same manner as the natural pericardium and may prevent infection from occupying the deep intrapericardial space, for example, sequestering an ascending aortic graft from a sternal wound infection.

Rationale for or against performing PCR: The functional goals of PCR can be ranked as follows (from highest to lowest in importance):

- Reestablish normal physiology

- Avoid blood collection in pericardial well

- Decrease adhesions/scarring for easier reentry [Boyd 2012; Duvernoy 1995; Matsumura 2008; Minale 1988]

Infection protection [Kaya 2015; Nandi 1976] and decreased inflammation were ranked as of lesser importance.

While prevention of adhesions may be important in the long term, especially if reoperation is eventually required, the short-term benefits of PCR seem to weigh more heavily for some surgeons. A collection of blood in the pericardial well can lead to inflammation, postoperative atrial fibrillation, and longer-term complications such as adhesions [Cannata 2013]. Avoiding such a blood collection can lead to better outcomes [Cannata 2013]. A return to normal physiology in the postoperative period is desirable and may lead to faster recovery. Though the overall risk of infection is low, a sternal wound 
infection is less likely to involve the heart if the pericardium is closed. Thus, pericardial closure can decrease morbidity from a sternal wound infection.

Physicians who reconstruct the pericardium typically do so to:

- Decrease adhesions [Dantas 2010; Duvernoy 1995; Matsumura 2008; Minale 1988]

- Prevent postoperative complications (e.g., postoperative atrial fibrillation or bleeding resulting in intervention or longer hospital stay) [Boyd 2010; Kaya 2015; Rego 2019]

- Decrease technical difficulty of reoperation [Cannata 2013; Nandi 1976]

- Protect the anterior epicardium and grafts

- Restore natural form and function of the heart and pericardium

- Prevent wound debris/blood from accumulating in the pericardium or contacting the epicardium [Kaya 2015]

Some surgeons choose to not reconstruct the pericardium because of:

- Little definitive clinical data supporting safety and efficacy of pericardial reconstruction

- Convention and/or lack of education on reconstruction technique

- Cost and/or reimbursement

- Time and/or effort

- Fear of complications (e.g., graft constriction [Asanza 1976; Boyd 2012], tamponade [Asanza 1976; Boyd 2012; Dantas 2010], calcification/fibrosis, retained effusion, compression of heart [Asanza 1976; Boyd 2012; Damen 1989; Dantas 2010; Daughters 1992; Hunter 1992; Jarvinen 1987]

- Patient-related reasons (e.g., possible reoperation within 24 hrs)

Some surgeons may not know or do not have confidence in the benefits of PCR. However, the establishment of a strong consensus among cardiothoracic surgeons with significant experience performing PCR should lead to greater acceptance and broader adoption of PCR by the cardiothoracic surgical community in addition to improved patient outcomes.

Important clinical data which supports PCR: Authors agree regarding which of the data supporting PCR are of most significance. These are listed in Table 3. (Table 3)

Results from the RECON Study: The RECON study [Rego 2019], published in 2019, was a prospective, multicenter study of 1420 patients. All patients underwent open cardiac surgery followed by reconstruction of the pericardium using extracellular matrix (ECM) graft derived from porcine small intestinal submucosa. The study included 923 patients who underwent coronary artery bypass graft (CABG) surgery and 436 patients who underwent valve surgery. Patient outcomes were compared with those of the Nationwide Readmissions Database (NRD). The study found statistically significant improvements in outcomes in RECON study patients in comparison with those in the NRD. Compared to those in the NRD, RECON patients who underwent CABG were less likely to suffer bleeding complications $(1.2 \%$ vs.
$2.9 \%)$ or pericardial effusion ( $0.2 \%$ vs. $2.2 \%)$. Patients who underwent valve surgery were less likely to develop pleural effusion $(3.1 \%$ vs. $13.0 \%)$ or pericardial effusion $(1.5 \%$ vs. $2.6 \%)$. Thirty-day readmission rates were significantly lower following either valve surgery (hazard ratio [HR], 0.34) or CABG (HR, 0.42).

Furthermore, the rate of post-CABG atrial fibrillation in the PCR cohort was $14.4 \%$ vs. $25.6 \%$ reported by the 2019 Society of Thoracic Surgeons (STS) Adult Cardiac Surgery Database [Bowdish 2020]. Among those who underwent valve surgery, the rate of postoperative atrial fibrillation was $27 \%$ in RECON patients compared with $29.4 \%$ to $42.9 \%$ reported by the 2019 STS Adult Cardiac Surgery Database [Bowdish 2020]. (In randomized clinical trials of amiodarone, POAF occurred in $33 \%$ of control patients undergoing cardiac surgery and in $19 \%$ to $20 \%$ of those treated prophylactically with amiodarone) [Arsenault 2013; Burgess 2006].

With respect to the outcomes of the RECON study, the positive results from the RECON study are mostly attributed to certain factors, which are listed (in no particular order of importance) in Table 3. RECON was an open-ended registry without true standardization of technique and/or endpoints. A standardized approach to PCR would improve the quality of research on outcomes.

The RECON study had several limitations. ECM use was not randomized, and the control group was a national database. The two studies had different data reporting structures. The NRD database did not include some comorbidities, and so these were not incorporated into the inverse probability of treatment weighting. The smaller sample size of the RECON valve repair/replacement cohort prevented further stratification into separate valve repair and replacement subgroups. In addition, the RECON study included only patients treated in the United States.

Potential indications for PCR are:

- For patients with a high likelihood of reoperation [Duvernoy 1995; Matsumura 2008; Minale 1988; Nandi 1976]

- To reduce incidence of postoperative atrial fibrillation [Boyd 2010; Kaya 2015; Rego 2019]

PCR may decrease length of hospital stay [Kaya 2015]; however, there is less evidence to support this indication.

For patients with a high likelihood of reoperation, PCR may increase the safety of reoperation [Nandi 1976] and reduce surgical adhesions [Duvernoy 1995; Matsumura 2008; Minale 1988]. Surgical adhesions are known to increase the risk of cardiac reoperation [Cannata 2013; Ferraris 2018; Kaneko 2012; Okuyama 1999; Walther 2005]. Several studies provide evidence that PCR, either primary or with use of a suitable pericardial substitute, preserves the posterior sternal table to cardiac distance [Rao 1999], minimizes intrapericardial adhesions [Duvernoy 1995; Matsumura 2008; Minale 1988], and leads to safer reoperations [Nandi 1976].

Some studies have shown that PCR may reduce the incidence of postoperative atrial fibrillation in patients undergoing cardiac surgery [Boyd 2010; Kaya 2015; Rego 2019]. Supportive evidence includes a retrospective study [Boyd 2010], a prospective study [Rego 2019], and a randomized controlled 
regarding surgical techniques to use or avoid in PCR are listed in Figure 1. (Figure 1) Upon opening the pericardium, surgical techniques that preserve tissue viability for reconstruction can be important, especially if planning use of regenerative patches, such as ECM. Attention must be paid to methods for opening the pericardium and techniques for reconstruction, such as use of native vs. patch graft, suturing technique, sizing of patch, and placement of chest tubes.

Physiologic considerations for PCR: After reconstructing the pericardium, the interaction between the heart and the pericardium is hypothesized to influence heart function in two ways:

\section{Form:}

- Maintenance of normal position and orientation within the mediastinum

Function:

- Serves as a barrier against infection [Boyd 2012]

- Reduces friction between the heart and surrounding structures [Boyd 2012]

- Adjusts the heart's structural response to physical stresses during the cardiac cycle

\section{Box 1. Patient conditions suggesting poor candidacy for $\mathrm{PCR}^{\mathrm{a}}$}

- Modulates ventricular filling and strongly modulates interdependence of the left ventricle (LV) and right ventricle (RV) [Boyd 2012]

- Enhances RV function

- Influences structural behavior of the ventricular septum and the LV and RV free walls

- Protects against heart deformation during ventricular diastole

Elements of the recommended PCR technique that are most critical to clinical success: The elements most critical for clinical success are displayed in Figure 1A.

Additional evidence needed to determine full benefits of PCR: In order to clarify the benefits of PCR, additional evidence, especially high-level evidence, regarding outcomes is needed. See Figure 1B and the outcomes listed in Box 2. (Box 2)

Important reasons why a standardized technique is needed: In our consensus opinion, a standardized PCR approach and procedure should be implemented uniformly across the surgical community because it will lead to:

- Better patient outcomes when pericardial reconstruction is performed

\author{
Lack of native pericardium \\ Large pericardial defects \\ Bleeding tendencies \\ Immunosuppression \\ Chest radiation \\ Lung surgery \\ Pericarditis \\ Cancer in proximity to the pericardium \\ Calcification of the pericardium \\ Constrictive pericarditis \\ Previous surgery where edges of the pericardium cannot be identified \\ Intraoperative factors relating to anatomy that prevent reconstruction \\ Multiple previous reoperations
}

a Listed in no specific priority order

Box 2. Further research needed

\footnotetext{
Reduction of postoperative atrial fibrillation

Reduction of postoperative bleeding

Reduction of ventilator/ICU stay time

Reduction of hospital stay and readmission rate

Lack of negative effect on cardiac hemodynamics

Improvement in long-term cardiac hemodynamics

Patient pain and quality of life scores
} 
- More reliable and consistent data to fully demonstrate patient benefits

- Training of surgical residents in the most effective techniques

- Cost containment

Use of a standardized approach should generate highquality data, which will give the surgical community the opportunity to continue improving the technique of PCR.

The concerns of not having a standardized approach/ technique to performing pericardial reconstruction include negative outcomes due to poor technique and not realizing optimal outcomes for patients-i.e., reduced complications, safer reoperations, and improved postoperative management. There also could be adverse effects on long-term RV function because of postoperative adherence of the RV to the posterior sternal table.

\section{DISCUSSION}

The group perceived a need to compile this expert opinion report for several reasons: To improve the limited understanding among clinicians of the potential benefits and risks of PCR; to address knowledge gaps which could influence clinical decision-making regarding PCR; and to improve patient outcomes and quality of patient care.

To date, understanding of the risks and benefits of PCR has been limited among clinicians due to lack of standardization of patient selection, PCR technique, and outcome assessments.

Important clinical data lending support to PCR is found in prior clinical studies. Two or more studies have shown evidence of decreased adhesions [Duvernoy 1995; Minale 1988; Nandi 1976], reduced incidence of postoperative atrial fibrillation [Boyd 2010; Kaya 2015; Rego 2019], fewer pericardial effusions [Kargar 2007; Kaya 2015; Rego 2019], and lack of adverse effects [Asanza 1976; Bhatnagar 1998; Dantas 2010; Quarti 2011]. At least one randomized or large observational study has shown reduced bleeding [Rego 2019], fewer readmissions [Rego 2019], greater distance from the epicardial surface to the posterior sternal table [Rao 1999], fewer pleural effusions [Rego 2019], reduced rate of lung infection [Kaya 2015], and reduced hospital and CCU length of stay [Kaya 2015]. The older 1976 Nandi study also found reduced rates of reoperation, fewer episodes of cardiac tamponade, and improved outcomes with reoperation [Nandi 1976]. Many of these studies have significant limitations, which lends support to the need for further high-quality research.

The scientific literature regarding PCR describes four major arguments against closure/reconstruction. These include: 1) perceived increase in risk of pericardial tamponade, 2) unfavorable hemodynamic response, 3) perceived risk of graft compression, and 4) epicardial reactions and formation of adhesions [Boyd 2012].

To date, no randomized controlled trials, case-control studies, or even case series studies have demonstrated an increase in pericardial tamponade following closure. A case series published in 1975 of 100 patients, who had primary pericardial closure with open heart surgery, found no instances of cardiac tamponade [Cunningham 1975]. Measurements of sump drainage found that most postoperative bleeding originated from outside the pericardium [Cunningham 1975]. On the contrary, an older study found that primary pericardial closure was protective against cardiac tamponade [Nandi 1976], and more recent studies evaluating reconstruction with pericardial substitutes have shown no increase in cardiac tamponade [Bhatnagar 1998; Boyd 2010; Rego 2019].

The adverse hemodynamic parameters have occurred only transiently without known long-term adverse effects and have only been documented with primary closure (with the patient's own pericardium) [Bittar 2005; Boyd 2012]. No such adverse effects have been documented in studies involving reconstruction with pericardial substitutes.

With regard to graft compression, pericardial substitutes, sutured to the native edges of the pericardium, can be adjusted to leave some space around the great vessels and any bypass grafts [Boyd 2012].

Epicardial reactions and adhesion formation, if any, vary considerably depending on the nature of the material used in the pericardial substitute [Boyd 2012].

Each of these perceived risks could be ameliorated by implementing the expert opinion recommendations in this document, notably those listed in Figure 1, such as taut but tension-free closure, circumferential running sutures, adequate drainage, and if patch use is indicated, use of patches that stimulate the formation of site-specific tissue in situ, such as natural (non-chemically cross-linked) ECM.

Evidence from additional high-quality randomized controlled trials also could lead to the development of standardized formal guidelines regarding PCR and best surgical techniques, which could improve patient outcomes.

Primary closure vs. patch reconstruction: The general consensus in the literature has been to perform primary closure if feasible; however, in many patients, primary closure is not possible, and it may not be appropriate in patients with preoperative impairment of $\mathrm{LV}$ function or cardiac output or in perioperative patients who need high preloads to maintain satisfactory cardiac output [Boyd 2012].

Options for pericardial patch: Many pericardial patch products are available. These include polytetrafluoroethylene (PTFE, also known as Gore-Tex®), glutaraldehyde treated xenografts, bioresorbable polymer films, and nonchemically cross-linked decellularized ECM [Cunningham 1975]. PTFE and glutaraldehyde treated xenografts (such as bovine pericardium crosslinked with glutaraldehyde for durability) have both been associated with intense epicardial scarring and calcification. Among bioresorbable polymer films, PGA (polyglycolic acid) mesh was more adhesive to the posterior sternum than PTFE. Several others have been studied, but most have been limited to preclinical investigations. One barrier film was approved for use in pediatric patients [Boyd 2012].

$\mathrm{ECM}$, or extracellular matrix, is a three-dimensional, tissue-specific network of both structural and functional 
components; it provides the mechanical framework for each tissue and organ and is a substrate for cell signaling [Brown 2014]. ECM consists of a diverse mix of structural and functional proteins, glycoproteins, glycosaminoglycans, and other molecules [Brown 2014]. ECM also acts as a niche for stem cell differentiation [Brown 2014]. Through a process known as "dynamic reciprocity," the ECM exerts effects upon cellular behavior and phenotype; in return, these cells create, degrade, and remodel the ECM [Brown 2014]. ECM-based materials can act as inductive templates for constructive remodeling-inducing de novo functional, site-appropriate tissue formation - and have been used in a variety of regenerative medicine approaches to tissue reconstruction [Brown 2014].

The ability of an ECM scaffold material to act as an inductive template for tissue remodeling is dependent upon thorough decellularization [Brown 2016]. A scaffold that has not been fully decellularized will contain remnant DNA, epitopes, and other cell debris; these molecules are known to promote an inflammatory or rejection-type response [Brown 2016].

Decellularized, non-chemically cross-linked ECM derived from porcine small intestinal submucosa lacks proinflammatory cellular components, has the advantage of downregulating the inflammatory response, and resists calcification. As a bioregenerative platform, it recruits the host's own stem cells and incorporates them into the matrix. The eventual result is bioresorption of the ECM and complete conversion into native host tissue [Boyd 2012].

Cross-linking has been used to enhance the strength and durability of ECM [Ma 2014]. However, chemical crosslinking reagents alter the ligand structure of the ECM; this prevents degradation and is associated with a foreign body reaction and encapsulation rather than constructive remodeling [Brown 2016].

The impact of surgical technique on patch remodeling: Surgical technique affects remodeling outcomes. In order to properly remodel, the bioscaffold must be attached circumferentially and preferably with a running non-absorbable suture. If the bioscaffold is merely tacked intermittently to the pericardial edge, remodeling will be adversely affected (Figure 1).

In conclusion, this group of experts believe that PCR confers numerous clinical benefits to patients undergoing open heart surgery. Immediate postoperative benefits include lower rates of (1) bleeding, (2) atrial fibrillation, (3) pericardial effusion, and (4) readmissions. Longer-term benefits may include fewer adhesions and improved clinical success if reoperation is required.

Primary closure is preferred if feasible; however, in most patients, primary closure is not possible, and in patients with preoperative impaired LV function or cardiac output, primary closure may not be appropriate because of its documented but short-term adverse effects on hemodynamic function. If native closure is not an option, the pericardium may be reconstructed with a patch. Non-chemically cross-linked (natural) ECMs are a safe and effective option as a pericardial patch. Those that stimulate the formation of site-appropriate tissue in situ, such as natural ECM, may have advantages-downregulation of the inflammatory response, resistance to calcification, and bioregenerative properties-in comparison with synthetic or chemically cross-linked biologic patch materials, both of which tend to promote inflammation.

In our experience as practicing cardiovascular surgeons, we have observed that certain techniques applied to PCR lead to positive clinical outcomes and wish to share our experience with the rest of the cardiovascular community. The hope is this document will assist physicians and surgeons in delivering the highest quality of care to their patients. More than one effective approach to performing pericardial reconstruction may exist, but this expert opinion report encompasses what we currently believe to be most effective based on our prior clinical experience with pericardial reconstruction.

Based on the available evidence and our experience, we endorse the adoption of pericardial reconstruction as standard of care in open heart surgery. We believe that PCR technique should be standardized, and we endorse careful consideration of a standardized PCR approach and procedure across the surgical community. Adoption of a standardized PCR technique should improve the quality of future studies and lead to more meaningful data. We also endorse inclusion of PCR as a data point in the STS database to facilitate further study, and continued research assessing outcomes of patients undergoing PCR after cardiac procedures using standardized approaches in a broad patient base.

\section{REFERENCES}

Arsenault KA, Yusuf AM, Crystal E, et al. 2013. Interventions for preventing post-operative atrial fibrillation in patients undergoing heart surgery. Cochrane Database Syst Rev. CD003611. Published 2013 Jan 31.

Asanza L, Rao G, Voleti C, Hartstein ML, Wisoff BG. 1976. Should the pericardium be closed after an open-heart operation? Ann Thorac Surg. 22(6):532-534.

Benedetto U, Gaudino MF, Dimagli A, et al. 2020. Postoperative Atrial fibrillation and long-term risk of stroke after isolated coronary artery bypass graft surgery. Circulation. 142:1320-1329.

Bessissow A, Khan J, Deveareaux P, Alvarez-Garcia J, Alonso-Coellos P. 2015. Postoperative atrial fibrillation in non-cardiac and cardiac surgery: an overview. J Thromb Haemost. 13(Suppl. 1):S304-S312.

Bhatnagar G, Fremes SE, Christakis GT, Goldman BS. 1998. Early results using an ePTFE membrane for pericardial closure following coronary bypass grafting. Published online. 190-193.

Bittar MN, Barnard JB, Khasati N, Richardson S, Raja SG. 2005. Should the pericardium be closed in patients undergoing cardiac surgery? Interact Cardiovasc Thorac Surg. 4(2):151-155.

Bowdish ME, D'Agostino RS, Thourani VH, et al. 2020. The Society of Thoracic Surgeons Adult Cardiac Surgery Database: 2020 update on outcomes and research. Ann Thorac Surg. 109(6):1646-1655.

Boyd W, Johnson WE III, Sultan P, Deering T, Matheny R. 2010. Pericardial reconstruction using an extracellular matrix implant correlates with reduced risk of postoperative atrial fibrillation in coronary artery bypass surgery patients. Heart Surg Forum. 13(5):E311-E316.

Boyd WD, Tyberg JV, Cox JL. 2012. A review of the current status of pericardial closure following cardiac surgery. Expert Rev Cardiovasc 
Ther. 10(9):1109-1118.

Brown BN, Badylak SF. 2014. Extracellular matrix as an inductive scaffold for functional tissue reconstruction. Transl Res. 163(4):268-285.

Brown BN, Badylak SF. 2016. Extracellular matrix as an inductive scaffold for functional tissue reconstruction. In: Laurence J, Baptista P, Atala A, Beusekom M Van, eds. Translating Regenerative Medicine to the Clinic. Elsevier Academic Press. 11-29.

Burgess DC, Kilborn MJ, Keech AC. 2006. Interventions for prevention of postoperative atrial fibrillation and its complications after cardiac surgery: a meta-analysis. Eur Heart J. 27(23):2846-2857.

Butt JH, Olesen JB, Gundlund A, Kumler T, Havers-Borgersen PSOE, Aagaard DT. 2019. Long-term thromboembolic risk in patients with postoperative atrial fibrillation after left-sided heart valve surgery. JAMA Cardiol. 4(11):1139-1147.

Cannata A, Petrella D, Russo CF, et al. 2013. Postsurgical Intrapericardial adhesions: Mechanisms of formation and prevention. Ann Thorac Surg. 95:1818-1826.

Cunningham J, Spencer F, Zeff R, Williams C, Cukingnan R, Mullin M. 1975. Influence of primary closure of the pericardium after open-heart surgery on the frequency of tamponade, postcardiotomy syndrome, and pulmonary complications. J Thorac Cardiovasc Surg. 70(1):119-125.

Damen J, Bolton D. 1989. Acute hemodynamic effects of pericardial closure in man. Acta Anaesthesiol Scand. 33(3):207-209.

Dantas CE, SÁ MP, Bastos ES, Magnanini MM. 2010. Pericardium closure after heart operations: A safe option? Brazilian J Cardiovasc Surg. 25(3):365-370.

Daughters GT, Frist WH, Alderman EL, Derby GC, Ingels NB, Miller DC. 1992. Effects of the pericardium on left ventricular diastolic filling and systolic performance early after cardiac operations. J Thorac Cardiovasc Surg. 104(4):1084-1091.

Duvernoy O, Malm T, Ramstrom J, Bowald S. 1995. A biodegradable patch used as a pericardial substitute after cardiac surgery: 6- and 24-Month evaluation with CT. Thorac Cardiovasc Surg. 43(5):271-274.

Ferraris VA. 2018. Pericardial adhesions and cardiac surgeons' nightmares. J Thorac Cardiovasc Surg. 156(4):1609-1610.

Greenberg JW, Lancaster TS, Schuessler RB, Melby SJ. 2017. Postoperative atrial fibrillation following cardiac surgery: a persistent complication. Eur J Cardiothorac Surg. 52(4):665-672.

Hunter S, Smith GH, Angelini GD. 1992. Adverse hemodynamic effects of pericardial closure soon after open heart operation. Ann Thorac Surg. 53(3):425-429.

Izzat M, Anderson M, Wilde P, Wisheart J, Bryan A, Angelini G. 1994. Hemodynamic effects and echocardiographic consequences of tensionfree pericardial closure after heart valve surgery. J Heart Valve Dis. 3(3):295-299.

Jarvinen A, Peltola K, Rasanen J, Heikkila J. 1987. Immediate hemodynamic effects of pericardial closure after open-heart surgery. Scand J Thorac Cardiovasc Surg. 21(2):131-134.

Kaneko Y, Hirata H, Achiwa I, Morishita H, Sotos H, Kobayashi J. 2012. Adhesion barrier reduces postoperative adhesions after cardiac surgery. Asian Cardiovasc Thorac Ann. 20(3):257-262.

Kargar F, Aazami MH. 2007. Rotational pericardial flap: An alternative tension-free technique for pericardial closure. J Thorac Cardiovasc Surg. 134(2):510-511.

Kaya M, Satilmisoglu M, Bugra A, et al. 2015. Impact of the total pericardial closure using bilateral trap door incision and pericardial cavity intervention on outcomes following coronary artery bypass grafting: a randomized, controlled, parallel-group prospective study. Interact Cardiovasc Thorac Surg. 21:727-733.

Lin MH, Kamel H, Singer DE, Su YL, Lee M, Ovbiagele B. 2019. Perioperative/postoperative atrial fibrillation and risk of subsequent stroke and/or mortality: A meta-analysis. Stroke. 50:1364-1371.

Ma B, Wang X, Wu C, Chang J. 2014. Crosslinking strategies for preparation of extracellular matrix-derived cardiovascular scaffolds. Regen Biomater. 1(1):81-89.

Matsumura G, Shin'Oka T, Ikada Y, Sakamoto T, H Kurosawa. 2008. Novel anti-adhesive pericardial substitute for multistage cardiac surgery. Asian Cardiovasc Thorac Ann. 16(4):309-312.

Minale C, Nikol S, Hollweg G, Mittermayer C, Messmer B. 1988. Clinical experience with expanded polytetrafluoroethylene Gore-Tex surgical membrane for pericardial closure: A study of 110 Cases. J Card Surg. 3(3):193-201.

Nandi P, Leung J, Cheung K. 1976. Closure of pericardium after open heart surgery: A way to prevent postoperative cardiac tamponade. $\mathrm{Br}$ Heart J. 38:1319-1323.

Okuyama N, Wang CY, Rose EA, et al. 1999. Reduction of retrosternal and pericardial adhesions with rapidly resorbable polymer films. Ann Thorac Surg. 68(3):913-918.

Quarti A, Nardone S, Colaneri M, Santoro G, Pozzi M. 2011. Preliminary experience in the use of an extracellular matrix to repair congenital heart diseases. Interact Cardiovasc Thorac Surg. 13(6):569-572.

Rao V, Komeda M, Weisel RD, Cohen G, Borger MA, David TE. 1999. Should the pericardium be closed routinely after heart operations? Ann Thorac Surg. 67(2):484-488.

Rego A, Cheung PC, Harris WJ, Brady KM, Newman J, Still R. 2019. Pericardial closure with extracellular matrix scaffold following cardiac surgery associated with a reduction of postoperative complications and 30-day hospital readmissions. J Cardiothorac Surg. 14(1):1-10.

Walther T, Rastan A, Dahnert I, et al. 2005. A novel adhesion barrier facilitates reoperations in complex congenital cardiac surgery. Surg Congenit Hear Dis. 129(2):359-363. 